\title{
Distress disclosure and psychological Distress among men: the role of feeling understood and loneliness
}

\author{
Brian TaeHyuk Keum ${ }^{1}$ (D) John L. Oliffe ${ }^{2} \cdot$ Simon M. Rice ${ }^{3} \cdot$ David Kealy $^{2} \cdot$ Zac E. Seidler $^{3} \cdot$ Dan W. Cox ${ }^{2} \cdot$ \\ Ronald F. Levant ${ }^{4}$ • John S. Ogrodniczuk ${ }^{2}$
}

Accepted: 25 July 2021

(C) The Author(s) 2021

\begin{abstract}
We examined whether men's distress disclosure would be indirectly associated with psychological distress through feeling understood by others and loneliness as serial or parallel mediators. We conducted path analyses $(N=1827$ adult men; mean 37.53 years, $S D=14.14$ ) to test the mediators while controlling for race/ethnicity. Post-hoc multi-group analysis was conducted to examine differences across White and Asian men. The serial mediation model fit the data better than the parallel mediation model. Controlling for race/ethnicity, a significant partial indirect association was found between greater distress disclosure and lower psychological distress through greater feelings of being understood and lower feelings of loneliness. Post-hoc multi-group analysis suggested that the hypothesized paths fit equivalently across White and Asian men, and the direct effect was not significant for Asian men. Our hypothesized serial model also fit the data significantly better than alternative models with distress and the mediators predicting disclosure. Distress disclosure may be a socially conducive strategy for men to feel understood and socially connected, a process that may be associated with lower psychological distress.
\end{abstract}

Keywords Distress disclosure $\cdot$ Feeling understood $\cdot$ Loneliness $\cdot$ Psychological distress $\cdot$ Mediation

Although effective mental health services are available, studies indicate that men are less likely than women to access such services (e.g., Seidler et al., 2016). Evidence suggests that adherence to traditional masculinity ideology may be a contributing factor, as men may conceal from others their psychological distress, believing that disclosing their problems would cause others to see them as "weak," "vulnerable," and "unmanly" (Addis \& Mahalik, 2003; Levant et al., 2013). Such self-stigmatizing beliefs have been found to dissuade psychological help-seeking among men, highlighting a paradox that men may only consider disclosing their issues for help as a last

Brian TaeHyuk Keum

briankeum@luskin.ucla.edu

1 Department of Social Welfare, University of California, Los Angeles, CA, USA

2 School of Nursing, University of British Columbia, Vancouver, Canada

3 Department of Nursing, University of Melbourne, Melbourne, Victoria, Australia

4 University of Akron, Akron, OH, USA resort and when their distress has reached critical levels (Levant et al., 2013; Vogel et al., 2011).

Accordingly, concealing distress has been associated with psychological health impairments such as depression (Kahn \& Garrison, 2009). Employing an interpersonal connectedness framework, Cox et al. (2020) reported that not feeling understood by others and loneliness sequentially (serial mediation) mediated the relationship between concealment of distress and symptoms of depression in a nationally representative sample of Canadian men. Men who endorsed higher distress concealment reported that they felt less understood by others, which in turn was associated with feeling lonelier, and ultimately contributed to greater depressive symptoms (Cox et al., 2020). Of note, Cox et al. (2020) reported that the role of feeling understood on depressive symptoms was explained through loneliness. It was suggested that men who feel less understood by others may feel detached and disconnected in their social realm, resulting in a sense of social isolation (Laurenceau et al., 1998) that may give rise to depressive symptoms (Cacioppo et al., 2010).

Findings from Cox et al. (2020) highlight distress concealment as an interpersonal and intrapersonal debilitating factor that contributes to greater depressive symptoms among men. On the other hand, the results also suggest that encouraging 
men to disclose their distress may promote greater social connectivity which may have psychological health benefits (Cox et al., 2020). As noted in the literature (e.g., Wei et al., 2005), although men who self-disclose their distress may feel vulnerable and that their masculine self-concept is threatened, doing so would allow an opportunity for others to listen, validate, empathize, and provide appropriate emotional and instrumental support for these men. This social connection likely facilitates feelings of being understood by others that may, in turn, mitigate a sense of loneliness among men. It is possible that the risk of psychological distress may be lower among men who feel less lonely as they are more comfortable sharing their distress that is reciprocated with understanding and acceptance from others (Cacioppo et al., 2010).

Thus, in contrast to worsening mental health associated with distress concealment (Cox et al., 2020; Kahn \& Hessling, 2001), we tested whether distress disclosure would be associated with lower psychological distress by way of enhancing one's feelings of being understood by others and reducing loneliness. As an active process of self-inhibition, concealment has been linked to psychological symptoms (e.g., anxiety, depression) that can develop from internalizing the ongoing distress (Kahn \& Hessling, 2001). As a related but distinctive process, disclosure involves an active sharing and confrontation of the distress, which in turn has been associated with lower psychological symptoms and physical health benefits (Kahn \& Hessling, 2001). Thus, the benefits of distress disclosure seem to depend on the basis that the disclosure process leads to a reduction in distress (Kahn \& Hessling, 2001). For example, while Cox et al. (2020) study found that distress concealment was ultimately associated with greater depression among men, one may question if disclosing the distress would have been associated with lower distress, thereby mitigating the onset of depressive symptoms.

Accordingly, extending the model reported in the Cox et al. (2020) study, we examined feeling understood and loneliness as mediators of the relationship between distress disclosure and psychological distress. In doing so, we examined if the sequential mediation previously proposed (Cox et al., 2020) or parallel mediation (another theoretically justifiable model) better explains the association between men's distress disclosure and psychological distress. For serial mediation, we expected that men who disclose their distress to others would report feeling understood by others, which likely reduces one's sense of feeling lonely. In turn, feeling less lonely may help minimize one's psychological distress. Our hypothesized serial mediation paths were: distress disclosure $\rightarrow$ feeling understood $\rightarrow$ reduced loneliness $\rightarrow$ lower psychological distress.

Alternatively, for parallel mediation, we expected concurrent mediation pathways through feeling understood or loneliness. Rather than feeling understood being the antecedent to loneliness, both may act as separate indirect pathways as the literature suggests some mutually exclusive processes.
For example, Lee and Ko (2018) suggest that people can still feel lonely even if they are socially interacting with others (e.g., understood by others). Thus, while disclosing distress may be associated with feeling understood by others, it can simultaneously be related to certain changes in loneliness based on whether, for example, the responses from others have met the expectation and the need of the person disclosing the distress (Lee \& Ko, 2018). Additionally, within the online space, people may have ample opportunities to disclose about themselves to feel understood by others but still feel lonely due to the lack of deeper human interaction (Hunt et al., 2018). Thus, we tested both pathways in our parallel mediation model: (a) distress disclosure $\rightarrow$ feeling understood $\rightarrow$ lower psychological distress, and (b) distress disclosure $\rightarrow$ reduced loneliness $\rightarrow$ lower psychological distress.

In our models, we controlled for race/ethnicity. The dominant narrative around masculine norms in Western societies generally discourages men from disclosing their distress and problems (Addis \& Mahalik, 2003; Levant et al., 2013). Yet, some indirect evidence suggests that racial/ethnic minority men may be less likely to self-disclose their problems compared to White men based on how they were socialized in their respective cultures (e.g., Heath et al., 2016; Zane \& Ku, 2014). For example, culture-specific factors, such as saving face and public reputation concerns, have been found to be particularly salient deterrents of distress disclosure among certain racial/ ethnic minority individuals including Asian Americans (Zane $\& \mathrm{Ku}, 2014$ ) and among Middle Eastern individuals (Heath et al., 2016). Furthermore, in comparison to Western ideals that emphasize individualism, the nature of loneliness may also have different meanings for certain racial/ethnic minority individuals who have been socialized with greater familyoriented and collectivistic values (e.g., Lykes \& Kemmelmeier, 2014). For instance, Lykes and Kemmelmeier (2014) found that the lack of family interactions was more predictive of loneliness for those in collectivistic societies whereas the lack of connections with friends and confidants was more closely associated with loneliness for those in individualistic societies. Thus, in the former case, even if men feel understood by others, whether they feel more connected or isolated may depend on the role (e.g., family vs. friends) and the importance of others providing such support. Given these potential racial differences, race/ethnicity was added as a covariate in our models.

\section{Methods}

\section{Participants and Procedures}

Data were collected from a non-probability sample of 1827 help-seeking adult men. The mean age was 37.53 ( $S D=$ 14.14). The majority of the men who participated in the survey 
resided in Canada $(42 \%, n=767)$, followed by $18 \%$ in the United Kingdom $(n=332), 16 \%$ in the United States $(n=284), 4 \%$ in Australia $(n=79)$, and $19 \%$ other $(n=355)$. About $72 \%$ of the sample identified as White $(n=1307), 13 \%$ as Asian $(n=245), 4 \%$ as Black $(n=72), 5 \%$ as Multiracial $(n=82), 2 \%$ as Indigenous $(n=37), 2 \%$ as Hispanic $(n=30)$, and $2 \%$ as other $(n=54)$. About $85 \%$ identified as heterosexual $(n=1561), 7 \%$ as gay $(n=136), 6 \%$ as bisexual $(n=104)$, and $2 \%$ as Other $(n=26)$. About $36 \%$ identified as single $(n=$ $655), 3 \%$ dating $(n=54), 23 \%$ in a committed relationship $(n=414), 31 \%$ married, $(n=567)$, and $7 \%$ divorced or separated $(n=137)$. The mean annual income among the participants was $\$ 30,349$. In terms of highest education achieved, $4 \%$ had less than high school education $(n=80), 19 \%$ completed high school education $(n=347), 22 \%$ had some college education but no degree $(n=396), 19 \%$ had a technical diploma or trade qualification $(n=346), 19 \%$ had an undergraduate degree $(n=343)$, and $17 \%$ had a graduate degree $(n=315)$.

Participants were recruited through the HeadsUpGuys website (https://headsupguys.org), a leading online resource that provides information about psychological services, and stories about other men's help-seeking and recovery stories on depression and suicide prevention (Ogrodniczuk et al., 2018). An advertisement of the study was placed on the website for men to provide their contact information if they were interested in participating in the study. Men interested in participating were provided a link to a Qualtrics hosted online survey where they were provided information on informed consent. Inclusion criteria were: (a) adult men aged 18 years or older, (b) able to read and understand English, and (c) having an internet connection to complete the survey. Those meeting the inclusion criteria and consenting to participate then proceeded with the survey. Following the survey, they were given an online debriefing. The Behavioral Research Ethics Board at the University of British Columbia (\#H1701334) provided ethics approval for the current study.

\section{Measures}

\section{Psychological Distress}

The Kessler-6 (K6) was used to measure general psychological distress (Kessler et al., 2003). The measure includes six questions and asks the frequency of psychological distress symptoms such as depression and anxiety in the past 30 days rated on a scale of 1 (none of the time) to 5 (all of the time). Items included how often they felt "hopeless", "so depressed that nothing could cheer them up", "worthless, nervous, restless or fidgety", and "that everything was an effort." Higher scores indicate higher levels of psychological distress symptoms and scores range from 6 to 30 . The scale has shown adequate reliability for Black, Asian, Latinx individuals (alpha $=.83$; Byrd, 2012; Prochaska et al., 2012). The Cronbach's alpha for the current sample was .87.

\section{Distress Disclosure}

The 12-item Distress Disclosure Index (DDI; Kahn \& Hessling, 2001) was used to assess participants' tendencies to share personally distressing information. The DDI contains items that assess disclosure behaviors (e.g., "When I feel upset, I usually confide in my friends") and concealment behaviors (e.g., "I prefer not to talk about my problems"). Participants rated their agreement with each item from 1 (strongly disagree) to 5 (strongly agree). The six concealment items were reverse-scored, and the responses were summed with higher scores indicating greater disclosure tendencies. Given that disclosure and concealment are likely to co-occur (Kahn \& Hessling, 2001), we combined the disclosure subscale scores and the reversed scores of the concealment subscale to capture the fuller breadth of the distress disclosure process (i.e., behavioral tendencies to disclose as well as to not conceal). Convergent validity of the DDI has been evidenced via associations with scores of relevant constructs (e.g., self-concealment, ambivalence over expression), and further supported in a multi-trait multi-method study (Kahn et al., 2012). The current sample Cronbach's alpha was .91.

\section{Loneliness}

Loneliness was measured using a single item ("I feel completely alone") rated on a 5-point scale from 1 (not at all) to 5 (extremely). Meta-analysis studies suggest that single-item measures of loneliness show high correlations with the UCLA Loneliness Scale and other similar constructs (Pinquart \& Sorensen, 2001).

\section{Feeling Understood}

Feeling understood was also assessed using a single item ("I feel deeply understood by at least one person in my life"), rated on a scale ranging from 1 (strongly disagree) to 5 (strongly agree). The single item was also used in Cox et al. (2020) study with good predictability of loneliness and psychological distress.

\section{Data Analysis}

To test our serial (Fig. 1) and parallel (Fig. 2) mediation models, we used Mplus 7.11 (Muthén \& Muthén, 2017) with maximum likelihood estimation. DDI was our predictor, feeling understood and loneliness were mediators, and K-6 was our outcome variable. In the serial mediation model, we tested feeling understood and loneliness as sequential mediators (DDI $\rightarrow$ feeling understood $\rightarrow$ loneliness $\rightarrow$ K-6). In the parallel mediation model, we tested each mediator in separate 
Fig. 1 Serial Mediation Model Depicting Indirect Relation of Distress Disclosure on Psychological Distress via Feeling Understood and Loneliness Note. $\mathrm{F}=$ Full sample; $\mathrm{W}=$ White men, $\mathrm{A}=$ Asian men Race/ethnicity covariate not depicted for brevity. ${ }^{* *} p<.01$, $* * * p<.001$.

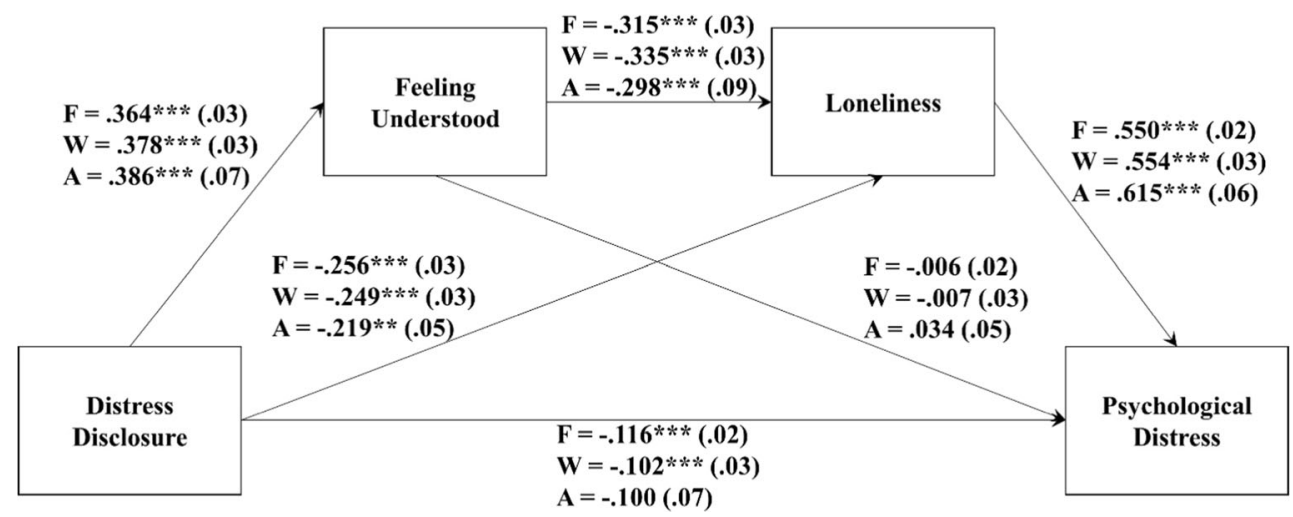

parallel paths to K-6 (DDI $\rightarrow$ feeling understood $\rightarrow$ K-6; $\mathrm{DDI} \rightarrow$ loneliness $\rightarrow \mathrm{K}-6$ ), with the two mediators correlated with each other. In both models, we regressed K-6 on race/ ethnicity as a covariate. We evaluated the model fit using the Yuan-Bentler (YB) scaled $\chi^{2}$ test and several approximate fit indices (Hu \& Bentler, 1999): (a) comparative fit index (CFI greater than .95 for good fit; .92 to .94 for adequate fit), (b) the standardized root mean square residual (SRMR; less than or equal to .08 for acceptable fit), (c) and the root mean square error of approximation (RMSEA; less than or equal to .08 for acceptable fit). Given that the serial model (less constrained) is nested in the parallel model (more constrained), we conducted a scaled chi-square difference test to assess the significant fit difference to identify the model to be retained.

To examine the specific path coefficients and the specific indirect (i.e., mediation) effects, we followed best practices (Hayes \& Scharkow, 2013) and adopted the bootstrap method with 10,000 random samples, which has the advantage of not assuming a normal distribution and using randomly generated samples to determine the standard errors, confidence intervals $(\mathrm{CI})$, and the significance of the indirect mediation effects more accurately. We used the $99 \%$ CI to examine the significance levels: if the $99 \%$ CI did not include a zero, the estimates were suggested to be significant at the $p<.01$ level.

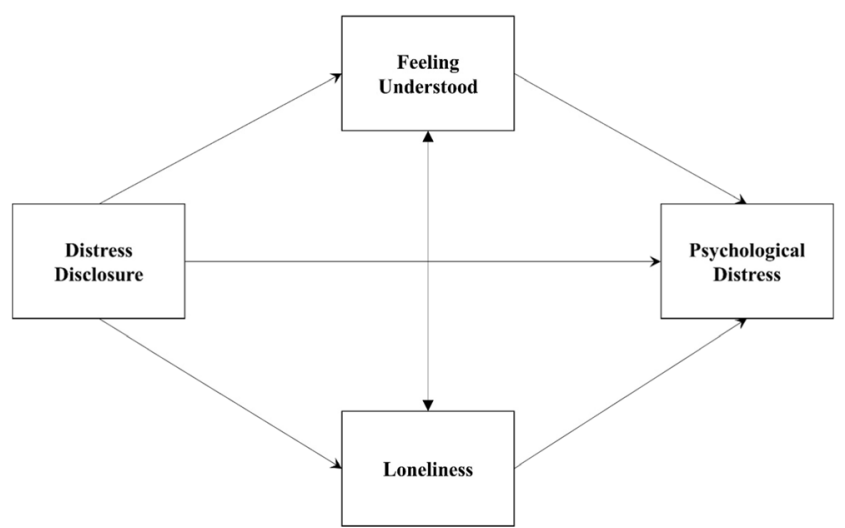

Fig. 2 Parallel Mediation Model Depicting Indirect Relation of Distress Disclosure on Psychological Distress via Feeling Understood and via Loneliness. Note. Double-head arrows indicate correlation. Race/ ethnicity covariate not depicted for brevity.

\section{Results}

Of the 1827 cases, 357 were missing more than $20 \%$ of the data and were removed. The remaining missing data ( 47 cases) were handled using full information maximum likelihood in Mplus 7.11. Prior to our analyses, we considered several potential demographic covariates (age, relationship status, and sexual orientation) relevant to our sample and the study variables. There is some emerging evidence that newer generations of men (e.g., millennials) may be adhering to a definition of masculinity that departs from traditional masculinity ideology (Debevec et al., 2013). Hence, we examined whether age was significantly correlated with our study variables. Additionally, we examined whether relationship status should be considered in our study as men who are in relationships (versus single men) may already have a partner they could confide in and share their distress. Age was not significantly associated with any of the predictor variables. We also did not observe any significant differences across the relationship status groups in our one-way ANOVA results. Hence, neither of these variables were considered further in the study. Bivariate correlations and descriptive statistics of the study variables are presented in Table 1 . The completely standardized path coefficient estimates are presented in Fig. 1, and the significant total direct, total indirect, and specific indirect effects are presented in Table 2.

\section{Path Analysis}

Overall model fit indices (Hu \& Bentler, 1999) suggested that our hypothesized parallel mediation model fit the data well: $\chi^{2}=$ 12.451, $d f=1, p=.002$, RMSEA $=.060$ [.031, .094]; $\mathrm{CFI}=.990 ; \mathrm{TLI}=.956 ; \mathrm{SRMR}=.018$. The hypothesized serial mediation model also fit the data well: $\chi^{2}=6.225, d f=2$, $p=.013$, RMSEA $=.060[.022, .109] ; \mathrm{CFI}=.995 ; \mathrm{TLI}=.956$; SRMR $=.018$. Satorra-Bentler scaled chi-square difference test suggested that there was a significant difference in model fit: $\Delta \chi^{2}=16.686, \Delta d f=1, p<.001$. This suggested that the model fit significantly worsened for the more constrained parallel mediation model compared to the less constrained serial mediation 
Table 1 Bivariate Correlations and descriptive statistics

\begin{tabular}{lllllll}
\hline Variables & 1 & 2 & 3 & $M$ & $S D$ & Range \\
\hline
\end{tabular}

\begin{tabular}{|c|c|c|c|c|c|c|}
\hline \multicolumn{7}{|c|}{ Full Sample } \\
\hline 1. Distress Disclosure & - & & & 24.45 & 9.38 & $8-50$ \\
\hline 2. Feeling Understood & $.36^{* *}$ & - & & 3.10 & 1.38 & $1-5$ \\
\hline 3. Loneliness & $-.37 * *$ & $-.41 * *$ & - & 3.34 & 1.27 & $1-5$ \\
\hline 4. Psychological Distress & $-.32 * *$ & $.27 * *$ & $.60 * *$ & 18.95 & 5.39 & $6-30$ \\
\hline \multicolumn{7}{|c|}{ Asian } \\
\hline 1. Distress Disclosure & - & & & 25.37 & 9.30 & $8-50$ \\
\hline 2. Feeling Understood & $.39 * *$ & - & & 3.26 & 1.38 & $1-5$ \\
\hline 3. Loneliness & $-.34 * *$ & $-.39 * *$ & - & 3.42 & 1.29 & $1-5$ \\
\hline 4. Psychological Distress & $-.29 * *$ & $-.24 * *$ & $.64 * *$ & 19.28 & 5.46 & $6-30$ \\
\hline \multicolumn{7}{|c|}{ White } \\
\hline 1. Distress Disclosure & - & & & 24.10 & 9.39 & $8-50$ \\
\hline 2. Feeling Understood & $.38 * *$ & - & & 3.04 & 1.38 & $1-5$ \\
\hline 3. Loneliness & $-.37 * *$ & $-.43 * *$ & - & 3.32 & 1.26 & $1-5$ \\
\hline 4. Psychological Distress & $-.31 * *$ & $-.28 * *$ & $.60 * *$ & 18.80 & 5.38 & $6-30$ \\
\hline
\end{tabular}

Note. $\mathrm{REM}=$ Racial/Ethnic Minority; $M=$ mean; $S D=$ Standard Deviation

$* p<.05, * * p<.01, * * * p<.001$

model. Thus, we retained the serial mediation model and proceeded to examine the indirect effects.

Overall, controlling for race/ethnicity, distress disclosure significantly predicted psychological distress (standardized effect $\beta=-.321,99 \%$ bootstrapped $\mathrm{CI}=[-.386,-.257])$. The total ef fect could be broken down into a significant direct effect $(\beta=$ $-.116,99 \%$ bootstrapped $\mathrm{CI}=[-.177,-.055])$ that accounted for $36 \%$ of the total effect, and significant total indirect effects through the hypothesized mediators (standardized total indirect effect $\beta=-.206,99 \%$ bootstrapped $\mathrm{CI}=[-.247,-.165])$ that accounted for the remaining $64 \%$ of the total effect. Regarding the specific indirect effects, feeling understood on its own was not a significant mediator of distress disclosure on psychological distress (standardized total indirect effect $\beta=-.002,99 \%$ bootstrapped $\mathrm{CI}=[-.026, .022]$ ), but loneliness (standardized total indirect effect $\beta=-.141,99 \%$ bootstrapped $\mathrm{CI}=[-.180$, $-.101]$ ) was a significant mediator. With both variables in the model, there was a significant indirect effect of distress disclosure on psychological distress through feeling understood and loneliness sequentially (standardized total indirect effect $\beta=-.116$, $99 \%$ bootstrapped $\mathrm{CI}=[-.177,-.055])$. These results suggested a partial serial mediation pattern.

\section{Post-hoc Multi-Group Analysis with White and Asian Men}

Considering the literature that pointed to potential cultural variation in how much men disclose and the psychological impact of disclosure to others, we controlled for race/ ethnicity in our main analysis. However, to assess potential group differences that are possible within our allotted sample sizes among racial groups, we conducted a post-hoc multigroup analysis of the serial mediation model across White $(n=1049)$ and Asian $(n=245)$ men. We were not able to examine comparisons with other groups due to a lack of sample sizes (e.g., Black, $n=72$; Multiracial, $n=82$; Hispanic, $n=30$ ). We tested the null hypothesis that the structural paths of the serial mediation model would fit equivalently across White and Asian men. Specifically, we compared an unconstrained model that allowed the structural paths to be freely estimated across the two groups, and a constrained model that fixed the corresponding structural paths to be the same across the two groups. Model fit indices showed that, for the constrained model, the overall model fit was adequate: $\chi^{2}=.957, d f=6, p=.987 ;$ RMSEA $=.000(\mathrm{CI}=[.000$, $.000]) ; \mathrm{CFI}=1.000$; SRMR $=.009$. The unconstrained model was saturated and just-identified: $\chi^{2}=0, d f=0$, RMSEA $=.000 ; \mathrm{CFI}=1.000$; $\mathrm{SRMR}=.000$. Because these two models were nested, we conducted a scaled chi-square difference test: $\Delta \chi^{2}=.957, \Delta d f=6, p=.987$. This indicated that the overall fit for the constrained model did not differ from the unconstrained model, meaning that when we fixed the structural paths to be equal across the two groups, the overall model fit did not worsen compared to the unconstrained model. This suggested that our hypothesized structural path model did not show significant differences between White and Asian men. Thus, our previous model estimates for the full sample applied equivalently to the two groups. Examining the mediation results (Table 2) indicated that the partial serial mediation found for the full sample was consistent for both groups, except that the direct effect was not significant in the Asian group. The Wald test of parameter constraints suggested that the indirect effects via feeling understood and loneliness were not significantly different between White and Asian men, $\chi^{2}(1)=.019, p=.890$.

\section{Post-hoc Comparisons with Alternative Models}

Although we found support for a hypothesized serial mediation model, we are not able to assume any causality due to the use of cross-sectional data. Hence, we further examined several alternative indirect pathways to assess whether the hypothesized serial model indeed fit the data best compared to the alternative models. First, we examined an alternative model in which distress disclosure could be predicted by loneliness and feeling understood, which, in turn, may be associated with lower distress. For example, in online settings, psychological well-being, interpersonal, and intrapersonal motivations were found to influence self-disclosure tendencies (Luo \& Hancock, 2020). This model (feeling understood $\rightarrow$ reduced loneliness $\rightarrow$ distress disclosure $\rightarrow$ lower psychological distress) also fit the data well and the fit indices were comparable to the hypothesized serial model: $\chi^{2}=11.357, d f=2, p=.003$, 
Table 2 Estimate of Indirect Effects from Bootstrap Analysis

\begin{tabular}{|c|c|c|c|c|c|}
\hline IV & Mediator(s) & DV & Standardized Effect Estimate & $S E$ & 99\% Bootstrapped CI \\
\hline \multicolumn{6}{|c|}{ Full Sample } \\
\hline \multicolumn{6}{|c|}{ Total Effect } \\
\hline DDI & & K6 & -.321 & .03 & {$[-.386,-.257]$} \\
\hline \multicolumn{6}{|c|}{ Total Direct Effect } \\
\hline DDI & & K6 & -.116 & .02 & {$[-.177,-.055]$} \\
\hline \multicolumn{6}{|c|}{ Total Indirect Effect } \\
\hline DDI & & K6 & -.206 & .02 & {$[-.247,-.165]$} \\
\hline \multicolumn{6}{|c|}{ Specific Indirect Effects } \\
\hline $\mathrm{DDI} \rightarrow$ & FeelUnd $\rightarrow$ & K6 & -.002 & .01 & {$[-.026, .022]$} \\
\hline $\mathrm{DDI} \rightarrow$ & Lone $\rightarrow$ & K6 & -.141 & .02 & {$[-.180,-.101]$} \\
\hline $\mathrm{DDI} \rightarrow$ & FeelUnd $\rightarrow$ Lone $\rightarrow$ & K6 & -.116 & .01 & {$[-.177,-.055]$} \\
\hline \multicolumn{6}{|c|}{ Asian } \\
\hline \multicolumn{6}{|c|}{ Total Effect } \\
\hline DDI & & K6 & -.293 & .08 & {$[-.509,-.076]$} \\
\hline \multicolumn{6}{|c|}{ Total Direct Effect } \\
\hline DDI & & K6 & -.100 & .07 & {$[-.289, .089]$} \\
\hline \multicolumn{6}{|c|}{ Total Indirect Effect } \\
\hline DDI & & K6 & -.193 & .05 & {$[-.332,-.053]$} \\
\hline \multicolumn{6}{|c|}{ Specific Indirect Effects } \\
\hline $\mathrm{DDI} \rightarrow$ & FeelUnd $\rightarrow$ & K6 & .013 & .03 & {$[-.064, .090]$} \\
\hline $\mathrm{DDI} \rightarrow$ & Lone $\rightarrow$ & K6 & -.135 & .05 & {$[-.274, .004]$} \\
\hline $\mathrm{DDI} \rightarrow$ & FeelUnd $\rightarrow$ Lone $\rightarrow$ & K6 & -.071 & .03 & {$[-.138,-.004]$} \\
\hline \multicolumn{6}{|c|}{ White } \\
\hline \multicolumn{6}{|c|}{ Total Effect } \\
\hline DDI & & K6 & -.313 & .08 & {$[-.389,-.237]$} \\
\hline \multicolumn{6}{|c|}{ Total Direct Effect } \\
\hline DDI & & K6 & -.102 & .07 & {$[-.174,-.029]$} \\
\hline \multicolumn{6}{|c|}{ Total Indirect Effect } \\
\hline DDI & & K6 & -.211 & .05 & {$[-.260,-.161]$} \\
\hline \multicolumn{6}{|c|}{ Specific Indirect Effects } \\
\hline DDI & FeelUnd $\rightarrow$ & K6 & -.003 & .03 & {$[-.032, .026]$} \\
\hline $\mathrm{DDI} \rightarrow$ & Lone $\rightarrow$ & K6 & -.138 & .05 & {$[-.186,-.090]$} \\
\hline $\mathrm{DDI} \rightarrow$ & FeelUnd $\rightarrow$ Lone $\rightarrow$ & K6 & -.070 & .03 & {$[-.093,-.047]$} \\
\hline
\end{tabular}

Note. DDI = Distress Disclosure Index; FeelUnd = Feeling Understood; Lone = Loneliness; K6 =

Psychological Distress; IV = Independent Variable; DV = Dependent Variable; $S E=$ Standard.

Error; $\mathrm{CI}=$ Confidence Interval. ${ }^{*} p<.05, * * p<.01$.
RMSEA $=.058[.029, .093] ;$ CFI $=.991 ;$ TLI $=.960 ;$ $\mathrm{SRMR}=.018 ; \mathrm{AIC}=22,031.28 ; \mathrm{BIC}=22,099.21$. However, the hypothesized serial mediation model had AIC and BIC values $(\mathrm{AIC}=17,170.22 ; \mathrm{BIC}=17,238.80)$ that were lower by more than 10 units, suggesting empirical support for a better fit (Burnham \& Anderson, 2004) compared to the alternative model.

Second, we also examined an alternative model based on potential reverse causality (Leszczensky \& Wolbring, 2019). Based on theory and empirical evidence (e.g., Cox et al., 2020), we theorized that distress disclosure would predict psychological distress. Although we have argued that traditional masculinity ideology and stigmatizing beliefs against helpseeking would make it difficult for men to share their distress (Levant et al., 2013; Vogel et al., 2011), it is also possible that psychological distress predicts distress disclosure: i.e., men who are experiencing high levels or critical levels of distress may be pushed to share their distress to others (Vogel et al., 2011). In turn, the disclosure could be associated with greater feelings of being understood and reduced feelings of loneliness. This model (psychological distress $\rightarrow$ distress disclosure $\rightarrow$ feeling understood $\rightarrow$ reduced loneliness) also fit the data well but the fit indices were slightly less adequate than the hypothesized serial model and the RMSEA value was 
approaching the minimum acceptable value of $.08: \chi^{2}=$ 15.857, $d f=2, p=<.01$, RMSEA $=.071[.041, .105]$; $\mathrm{CFI}=.987 ; \mathrm{TLI}=.942 ; \mathrm{SRMR}=.025 ; \mathrm{AIC}=22,258.62$; $\mathrm{BIC}=22,326.55$. Furthermore, the AIC and BIC values were also higher by more than 10 values compared to the hypothesized serial model. Thus, overall, the hypothesized serial model demonstrated a better fit to the data than the alternative model.

\section{Discussion}

The current study examined a mediation model as to whether distress disclosure among men may lead to lower psychological distress (as measured by the K-6), extending the Cox et al. (2020) study which tested the indirect effects of feeling understood and loneliness on depressive symptoms (as measured by the PHQ-9; Kroenke et al., 2001). Controlling for race/ethnicity, we found a significant partial indirect association between greater distress disclosure and lower psychological distress through greater feelings of being understood and lower feelings of loneliness. Contributing to theory building, our model testing suggested that feeling understood and loneliness likely function as serial mediators (as reported in Cox et al., 2020) rather than as parallel mediators. Results suggested a potential sequential flow of the two intermediary variables as the impact of disclosure and feeling understood was explained through loneliness. Post-hoc multi-group analysis between White and Asian men suggested that our hypothesized serial mediation model functioned equivalently. Interestingly, the direct effect of distress disclosure was not significant among Asian men, suggesting that the mediating roles of feeling understood and reduction in loneliness may be particularly salient on the lower psychological distress associated with distress disclosure for these men. Finally, the hypothesized serial mediation model fit the data better than an alternative model with feeling understood and loneliness as predictors of distress disclosure and a model with psychological distress predicting distress disclosure.

In line with the findings of Cox et al. (2020), the results of the present study point to distress disclosure as a socially conducive strategy for men to feel understood, accepted, and connected to others in their social realm. Reflecting the plethora of benefits associated with greater social support and connectedness (Santini et al., 2015), we found that this pathway was associated with lower psychological distress, highlighting facilitation of distress disclosure as a potential prevention and intervention mechanism to promote greater social and psychological well-being among men. One important note to be made, however, is that this process may not be so straightforward; for many men, they may choose not to disclose their distress to others due to prior negative experiences from doing so. A recent transnational survey (United Kingdom, Australia,
United States, Canada) found that $41 \%$ of men reported regretting sharing to others about their problems and over half of these men reported that this experience prevented them from disclosing their problems again (Movember, 2019). Disclosure would also involve significant vulnerability and threats to their masculine self-concept, especially for men who strongly endorse traditional masculinity ideology (Levant et al., 2013). Thus, it is likely that encouraging men to open up about their problems should be done within a supportive and male-sensitive context that can allow men to process varying reactions (negative and positive) from others and prevent negative internalization. Ultimately, with increasing comfort and familiarity in sharing their distress with others, this process may gradually allow men to eventually seek more professional help regarding their problems and issues, including mental health services (Levant et al., 2013; Vogel et al., 2011).

Given that the racial/ethnic minority men in our study reside in Western societies that emphasize the dominant narrative around hegemonic masculinity ideals (Addis \& Mahalik, 2003; Levant et al., 2013), the findings may reflect the general conformity to these ideals that likely influence both White and racial/ethnic minority men's daily negotiations on whether to disclose their distress to others or refrain from doing so in order to align with the dominant masculine norms. However, our post-hoc multi-group analysis showed some nuanced differences in this process for Asian men in comparison to White men. Whereas the mediation was partial for White men, the lack of direct effect for Asian men suggested that the hypothesized mechanism may have greater meaning among Asian men. Asian men may face culture-specific barriers such as "saving face" that discourage them from opening up about their problems with others (Zane \& Ku, 2014). However, when they do disclose, it may be with close relatives (e.g., family members) who may be able to provide a more positive and committed interaction to hearing out their problems compared to disclosing to others such as friends and peers whose reactions may vary and have less meaning depending on the relationship (Lykes \& Kemmelmeier, 2014). In this context, the role of feeling understood may have greater salience and potency in reducing loneliness, which can help mitigate their distress. Collectively, our results suggest the importance of both shared and racial group-specific factors that may influence the distress disclosure process for men.

The strength of this study is the use of a large multiethnic sample and significant results based on the $99 \%$ CIs. However, there are several limitations that should be addressed in future studies. First, even though we tested a mediational model, the results were based on cross-sectional data. Hence, we are limited in what we can say about the directionality and causality of the variables. Future studies should employ longitudinal data to provide evidence on the directional and temporal mechanism of the model. Additionally, one 
might wonder if psychological distress could predispose men's disclosure of their distress. Although theory and empirical evidence on traditional masculinity ideology (Addis \& Mahalik, 2003; Levant et al., 2013) suggest that men are less willing to disclose their distress on their own, there may be promotive mechanisms that future studies can examine. This would be an important antecedent process to the disclosure mechanism we examined. Second, self-selection bias may have affected our results, as participants who visited the website and participated in the survey were likely help seeking regarding their current distress. For example, more than half (57\%) of our participants endorsed clinically significant psychological distress levels (K6 score $\geq 19$; Kessler et al., 2003). Given this, it is possible that our sample and the findings may have been more likely to reflect the hypothesized model since these men may have been more ready to share their distress with others to seek help. Thus, replication of our findings with non-help seeking men would be warranted. Third, our data were based on self-report measures that limit understanding of the objective aspects of how others were influential on men's feelings of being understood as well as their perceived loneliness. For instance, there may be discrepancies between actual support systems (e.g., greater or fewer close friends) and perceptions of social support (e.g., satisfaction with peer relationships) that may differentiate the usefulness of distress disclosure. Relatedly, loneliness and feeling understood were measured with single items, and future studies may need to corroborate our findings using more psychometrically rigorous measures. Fourth, caution must be used in interpreting and generalizing our findings to racial minority men. Although we controlled for race/ethnicity and assessed initial comparisons between White and Asian men, the overall results are based on a majority White sample. Furthermore, we were only able to compare the model across White and Asian men due to sample size limitations and little can be said about the generalizability toward other racial minority groups. For example, Jacoby et al. (2020) found that the majority of the Black men suffering from psychological symptoms in their study reported experiencing interpersonal and systemic barriers such as financial constraints, limited access to mental health care services, and fear of judgement from mental health professionals. Thus, there may be culture-specific mechanisms and barriers to disclosing distress in this group that warrants testing of our model. Future studies should replicate and extend the model with representative samples of racial minority men and assess further specific differences across racial groups. Culture-specific factors such as saving face and shame responses due to self-disclosure may be explored to gain a more culturally-relevant insight on benefits associated with distress disclosure among racial/ethnic minority men.
Finally, other salient identities such as sexual orientation must be considered in future studies. For example, whereas traditional masculinity ideology may be a predominant barrier for disclosing distress among heterosexual men (Levant et al., 2013; Vogel et al., 2011), this process may be further complicated for sexual minority men who also experience barriers related to minority stress and marginalization due to their sexual minority identities (Durso \& Meyer, 2013). Factors such as Anti-LGBTQ discrimination, stigma, and perceived lack of social support on LGBTQ issues may make it much more difficult for sexual minority men to willingly share their distress, particularly if the distress is tied to their sexual minority identities (Durso \& Meyer, 2013; Fingerhut et al., 2010). Hence, future studies should test whether the hypothesized model functions similarly among sexual minority men given these additional barriers.

In conclusion, the findings suggest that men who engage in disclosing their distress to others may feel more understood and less lonely, which likely contributes to better psychological well-being. Our initial inquiry into racial differences in this process suggested that the model may apply equivalently across White and Asian men despite evidence on cultural differences in distress disclosure and loneliness. Based on our findings, future studies should examine male-sensitive interventions or promotive factors that help to remove barriers for men from disclosing their distress to others. Additional theory building should also be conducted, especially on the application of the model with other racial minority men not examined in this study and other salient identities such as sexual orientation.

Availability of Data and Material (Data Transparency) Not applicable.

Code Availability (Software Application or Custom Code) Not applicable.

\section{Declarations}

Conflicts of Interest/Competing Interests The authors declare that they have no conflict of interest or competing interest.

Ethics Approval (Include Appropriate Approvals or Waivers) Ethics approval for the study was granted by the Behavioral Research Ethics Board at the University of British Columbia (\#H17-01334).

Consent to Participate (Include Appropriate Statements) All participants were provided informed consent and consented to participated in the study.

Consent for Publication (Include Appropriate Statements) All participants were provided informed consent that data will be used for publication. 
Open Access This article is licensed under a Creative Commons Attribution 4.0 International License, which permits use, sharing, adaptation, distribution and reproduction in any medium or format, as long as you give appropriate credit to the original author(s) and the source, provide a link to the Creative Commons licence, and indicate if changes were made. The images or other third party material in this article are included in the article's Creative Commons licence, unless indicated otherwise in a credit line to the material. If material is not included in the article's Creative Commons licence and your intended use is not permitted by statutory regulation or exceeds the permitted use, you will need to obtain permission directly from the copyright holder. To view a copy of this licence, visit http://creativecommons.org/licenses/by/4.0/.

\section{References}

Addis, M. E., \& Mahalik, J. R. (2003). Men, masculinity, and the contexts of help seeking. American Psychologist, 58(1), 5-14. https://doi.org/ 10.1037/0003-066X.58.1.5

Burnham, K. P., \& Anderson, D. R. (2004). Multimodel inference understanding AIC and BIC in model selection. Sociological Methods \& Research, 33, 261-304. https://doi.org/10.1177/0049124104268644

Byrd, D. R. (2012). Race/ethnicity and self-reported levels of discrimination and Psychological Distress, California, 2005. Preventing Chronic Disease, 9(5), E156. https://doi.org/10.5888/pcd9.120042

Cacioppo, J. T., Hawkley, L. C., \& Thisted, R. A. (2010). Perceived social isolation makes me. sad: 5-year cross-lagged analyses of loneliness and depressive symptomatology in the Chicago Health, Aging, and Social Relations Study. Psychology and Aging, 25(2), 453-463. https://doi.org/10.1037/a0017216

Cox, D. W., Ogrodniczuk, J. S., Oliffe, J. L., Kealy, D., Rice, S. M., \& Kahn, J. H. (2020). Distress concealment and depression symptoms in a national sample of Canadian men: Feeling understood and loneliness as sequential mediators. The Journal of Nervous and Mental Disease, 208(6), 510-513. https://doi.org/10.1097/NMD.0000000000001153

Debevec, K., Schewe, C. D., Madden, T. J., \& Diamond, W. D. (2013). Are today's millennials splintering into a new generational cohort? Maybe! Journal of Consumer Behaviour, 12(1), 20-31. https://doi. org/10.1002/cb.1400

Durso, L. E., \& Meyer, I. H. (2013). Patterns and predictors of disclosure of sexual orientation to healthcare providers among lesbians, gay men, and bisexuals. Sexuality Research and Social Policy, 10(1), 35-42. https://doi.org/10.1007/s13178-012-0105-2

Fingerhut, A. W., Peplau, L. A., \& Gable, S. L. (2010). Identity, minority stress and psychological well-being among gay men and lesbians. Psychology \& Sexuality, 1(2), 101-114. https://doi.org/10.1080/ 19419899.2010.484592

Hayes, A. F., \& Scharkow, M. (2013). The relative trustworthiness of inferential tests of the indirect effect in statistical mediation analysis: Does method really matter? Psychological science, 24(10), 19181927. https://doi.org/10.1177/0956797613480187

Heath, P. J., Vogel, D. L., \& Al-Darmaki, F. R. (2016). Help-seeking attitudes of united Arab Emirates students: Examining loss of face, stigma, and self-disclosure. The Counseling Psychologist, 44(3), 331-352. https://doi.org/10.1177/0011000015621149

Hu, L. T., \& Bentler, P. M. (1999). Cutoff criteria for fit indexes in covariance structure analysis: Conventional criteria versus new alternatives. Structural equation modeling: a multidisciplinary journal, 6(1), 1-55. https://doi.org/10.1080/10705519909540118
Hunt, M. G., Marx, R., Lipson, C., \& Young, J. (2018). No more FOMO: Limiting social media decreases loneliness and depression. Journal of Social and Clinical Psychology, 37(10), 751-768. https://doi.org/ 10.1521/jscp.2018.37.10.751

Jacoby, S. F., Rich, J. A., Webster, J. L., \& Richmond, T. S. (2020). Sharing things with people that I don't even know': help-seeking for psychological symptoms in injured Black men in Philadelphia. Ethnicity \& health, 25(6), 777-795. https://doi.org/10.1080/ 13557858.2018.1455811

Kahn, J. H., \& Garrison, A. M. (2009). Emotional self-disclosure and emotional avoidance: Relations with symptoms of depression and anxiety. Journal of Counseling Psychology, 56(4), 573-584. https:// doi.org/10.1037/a0016574

Kahn, J. H., \& Hessling, R. M. (2001). Measuring the tendency to conceal versus disclose psychological distress. Journal of Social and Clinical Psychology, 20(1), 41-65. https://doi.org/10.1521/jscp.20.1.41.22254

Kahn, J. H., Hucke, B. E., Bradley, A. M., Glinski, A. J., \& Malak, B. L. (2012). The Distress Disclosure Index: A research review and multitrait-multimethod examination. Journal of Counseling Psychology, 59(1), 134-149. https://doi.org/10.1037/a0025716

Kessler, R. C., Berglund, P., Demler, O., Jin, R., Koretz, D., Merikangas, K. R., et al. (2003). The epidemiology of major depressive disorder: results from the National Comorbidity Survey Replication (NCS-R). Jama, 289(23), 3095-3105. https://doi.org/10.1001/jama.289.23.3095

Kroenke, K., Spitzer, R. L., \& Williams, J. B. (2001). The PHQ-9: validity of a brief depression severity measure. Journal of general internal medicine, 16(9), 60-613. https://doi.org/10.1046/j.1525-1497. 2001.016009606.x

Laurenceau, J.-P., Barrett, L. F., \& Pietromonaco, P. R. (1998). Intimacy as an interpersonal process: The importance of self-disclosure, partner disclosure, and perceived partner responsiveness in interpersonal exchanges. Journal of Personality and Social Psychology, 74(5), 1238-1251. https://doi.org/10.1037/0022-3514.74.5.1238

Lee, Y., \& Ko, Y. G. (2018). Feeling lonely when not socially isolated: Social isolation moderates the association between loneliness and daily social interaction. Journal of Social and Personal Relationships, 35(10), 1340-1355. https://doi.org/10.1177/ 0265407517712902

Leszczensky, L., \& Wolbring, T. (2019). How to deal with reverse causality using panel data? Recommendations for researchers based on a simulation study. Sociological Methods \& Research, 1-29. doi: https://doi.org/10.1177/0049124119882473

Levant, R. F., Hall, R. J., \& Rankin, T. J. (2013). Male Role Norms Inventory-Short Form (MRNI-SF): Development, confirmatory factor analytic investigation of structure, and measurement invariance across gender. Journal of Counseling Psychology, 60(2), 228238. https://doi.org/10.1037/a0031545

Luo, M., \& Hancock, J. T. (2020). Self-disclosure and social media: motivations, mechanisms and psychological well-being. Current Opinion in Psychology, 31, 110-115. https://doi.org/10.1016/j. copsyc.2019.08.019

Lykes, V. A., \& Kemmelmeier, M. (2014). What predicts loneliness? Cultural difference between individualistic and collectivistic societies in Europe. Journal of Cross-Cultural Psychology, 45(3), 468490. https://doi.org/10.1177/0022022113509881

Movember (2019). Perceptions of masculinity \& the challenges of opening up https://cdn.movember.com/uploads/images/2012/News/ UK\% 20IRE\%20ZA/Movember\%20Masculinity \%20\%26\% 20Opening\%20Up\%20Report\%2008.10.19\%20FINAL.pdf

Muthén, L. K., \& Muthén, B. O. (2017). 1998-2017. Mplus user's guide. Muthén \& Muthén. 
Ogrodniczuk, J., Oliffe, J., \& Beharry, J. (2018). HeadsUpGuys: Canadian online resource for men with depression. Canadian Family Physician, 64(2), 93-94.

Pinquart, M., \& Sorensen, S. (2001). Influences on loneliness in older adults: A meta-analysis. Basic and applied social psychology, 23(4), 245-266. https://doi.org/10.1207/S15324834BASP2304 2

Prochaska, J. J., Sung, H. Y., Max, W., Shi, Y., \& Ong, M. (2012). Validity study of the K6 scale as a measure of moderate mental distress based on mental health treatment need and utilization. International journal of methods in psychiatric research, 21(2), 88-97. https://doi.org/10.1002/mpr.1349

Santini, Z. I., Koyanagi, A., Tyrovolas, S., Mason, C., \& Haro, J. M. (2015). The association between social relationships and depression: a systematic review. Journal of affective disorders, 175, 53-65. https://doi.org/10.1016/j.jad.2014.12.049

Seidler, Z. E., Dawes, A. J., Rice, S. M., Oliffe, J. L., \& Dhillon, H. M. (2016). The role of masculinity in men's help-seeking for depression: A systematic review. Clinical psychology review, 49, 106118. https://doi.org/10.1016/j.cpr.2016.09.002
Vogel, D. L., Heimerdinger-Edwards, S. R., Hammer, J. H., \& Hubbard, A. (2011). "Boys don't cry": Examination of the links between endorsement of masculine norms, self-stigma, and help-seeking attitudes for men from diverse backgrounds. Journal of Counseling Psychology, 58(3), 368-382. https://doi.org/10.1037/a0023688

Wei, M., Russell, D. W., \& Zakalik, R. A. (2005). Adult attachment, social self-efficacy, self-disclosure, loneliness, and subsequent depression for freshman college students: A longitudinal study. Journal of Counseling Psychology, 52(4), 602-614. https://doi. org/10.1037/0022-0167.52.4.602

Zane, N., \& Ku, H. (2014). Effects of ethnic match, gender match, acculturation, cultural identity, and face concern on self-disclosure in counseling for Asian Americans. Asian American Journal of Psychology, 5(1), 66-74. https://doi.org/10.1037/a0036078

Publisher's Note Springer Nature remains neutral with regard to jurisdictional claims in published maps and institutional affiliations. 